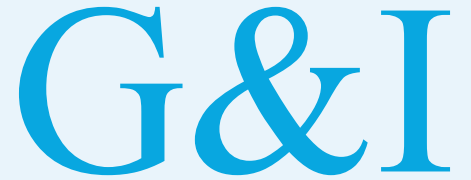

Genomics \& INFORMatics

\section{Original article}

eISSN 2234-0742

Genomics Inform 2021;19(4):e38

https://doi.org/10.5808/gi.21046

Received: August 19, 2021

Revised: September 27, 2021

Accepted: October 5, 2021

*Corresponding author:

E-mail: cookie_jklee@hotmail.com

Jeong Jin Yu, Gi Young Jang, and JongKeuk Lee contributed equally to this work.

\title{
Identification of rare coding variants associated with Kawasaki disease by whole exome sequencing
}

Jae-Jung Kim ${ }^{1}$, Young Mi Hong ${ }^{2}$, Sin Weon Yun ${ }^{3}$, Kyung-Yil Lee ${ }^{4}$, Kyung Lim Yoon ${ }^{5}$, Myung-Ki Han ${ }^{6}$, Gi Beom Kim ${ }^{7}$, Hong-Ryang Kil ${ }^{8}$, Min Seob Song ${ }^{9}$, Hyoung Doo Lee ${ }^{10}$, Kee Soo Ha ${ }^{11}$, Hyun Ok Jun ${ }^{12}$, Byung-Ok Choi ${ }^{13}$, Yeon-Mok Oh${ }^{14}$, Jeong Jin $\mathrm{Yu}^{15}$, Gi Young Jang ${ }^{11,16}$, Jong-Keuk Lee ${ }^{1 *}$, The Korean Kawasaki Disease Genetics Consortium

${ }^{1}$ Asan Institute for Life Sciences, Asan Medical Center, University of Ulsan College of Medicine, Seoul 05505, Korea

${ }^{2}$ Department of Pediatrics, Ewha Womans University Hospital, Seoul 07985, Korea

${ }^{3}$ Department of Pediatrics, Chung-Ang University Hospital, Seoul 06973, Korea

${ }^{4}$ Department of Pediatrics, Daejeon St. Mary's Hospital, College of Medicine, The Catholic University of Korea, Daejeon 34943, Korea

${ }^{5}$ Department of Pediatrics, Kyung Hee University Hospital at Gangdong, Seoul 05278, Korea

${ }^{6}$ Department of Pediatrics, Gangneung Asan Hospital, University of Ulsan College of Medicine, Gangneung 25440, Korea

${ }^{7}$ Department of Pediatrics, Seoul National University Children's Hospital, Seoul 03080, Korea

${ }^{8}$ Department of Pediatrics, Chungnam National University Hospital, Daejeon 35015, Korea

${ }^{9}$ Department of Pediatrics, Inje University Paik Hospital, Busan 47392, Korea

${ }^{10}$ Department of Pediatrics, Pusan National University Hospital, Busan 49241, Korea

${ }^{11}$ Department of Pediatrics, Korea University Guro Hospital, Seoul 08308, Korea

${ }^{12}$ Department of Pediatrics and Adolescent Medicine, Myongji Hospital, Goyang 10475, Korea

${ }^{13}$ Department of Neurology, Samsung Medical Center, Sungkyunkwan University School of Medicine, Seoul 04401, Korea

${ }^{14}$ Department of Pulmonary and Critical Care Medicine, Asan Medical Center, University of Ulsan College of Medicine, Seoul 05505, Korea

${ }^{15}$ Department of Pediatrics, Asan Medical Center, University of Ulsan College of Medicine, Seoul 05505, Korea

${ }^{16}$ Department of Pediatrics, Korea University Ansan Hospital, Ansan 15355, Korea

Kawasaki disease (KD) is an acute pediatric vasculitis that affects genetically susceptible infants and children. To identify coding variants that influence susceptibility to KD, we conducted whole exome sequencing of 159 patients with KD and 902 controls, and performed a replication study in an independent 586 cases and 732 controls. We identified five rare coding variants in five genes (FCRLA, PTGER4, IL17F, CARD11, and SIGLEC10) associated with $\mathrm{KD}$ (odds ratio [OR], 1.18 to $4.41 ; \mathrm{p}=0.0027-0.031$ ). We also performed association analysis in $26 \mathrm{KD}$ patients with coronary artery aneurysms (CAAs; diameter $>5$ $\mathrm{mm}$ ) and 124 patients without CAAs (diameter $<3 \mathrm{~mm}$ ), and identified another five rare coding variants in five genes (FGFR4, IL31RA, FNDC1, MMP8, and FOXN1), which may be associated with CAA (OR, 3.89 to $37.3 ; p=0.0058-0.0261)$. These results provide insights into new candidate genes and genetic variants potentially involved in the development of $\mathrm{KD}$ and $\mathrm{CAA}$.

Keywords: association study, coronary artery aneurysms, Kawasaki disease, whole exome sequencing 


\section{Introduction}

Kawasaki disease $(\mathrm{KD})$ is an acute, self-limited vasculitis that occurs predominantly in infants and young children [1]. $\mathrm{KD}$ is often complicated by coronary artery aneurysms (CAAs), which occur in approximately $15 \%-25 \%$ of untreated children and $3 \%-5 \%$ of children treated with high-dose intravenous immunoglobulin (IVIG) [2-4]. Although the pathogenesis of KD remains unknown, it has been postulated that $\mathrm{KD}$ is an abnormal immunological reaction to an infection in genetically susceptible individuals $[5,6]$.

Many complex traits are shaped by intricate interplay between common and rare genetic variants. Recently, genome-wide association studies (GWAS) of common single nucleotide polymorphisms (SNPs) have identified several variants associated with susceptibility to $\mathrm{KD}$ [7-11]. Although identification of these common variants has contributed somewhat to understanding the biology of $\mathrm{KD}$, many complexities of the disease remain to be unraveled [12]. Furthermore, most GWAS-derived SNPs do not directly influence the disease trait. Rather, they are index markers in linkage disequilibrium with causative variants for the disease; hence, it is necessary to identify rare coding variants that affect susceptibility to $\mathrm{KD}$ using other approaches, such as next-generation sequencing technology. Whole exome sequencing (WES) is a powerful technique for identification of rare protein-coding variants. Here, we used WES and association analysis to identify the protein-coding variants that influence susceptibility to $\mathrm{KD}$ and CAA.

\section{Methods}

\section{Subjects}

Children with $\mathrm{KD}$ were recruited from 12 tertiary academic hospitals in Korea, all of which participated in the Korean Kawasaki Disease Genetics Consortium. All patients were diagnosed by pediatricians in accordance with the diagnostic criteria of the American Heart Association $[13,14]$. All patients received a single highdose of IVIG $(2 \mathrm{~g} / \mathrm{kg})$. Two-dimensional echocardiography results were interpreted by pediatric cardiologists, and coronary arteries were categorized as normal or abnormal (showing coronary artery lesions (i.e., dilation or aneurysm)). A total of 200 children with KD were initially included in the WES analysis. Our WES data were deposited to the Biobank for Health Sciences, Chungwon, Korea (http:// nih.go.kr/biobank; accession number 2016-ER7401-00). For association analysis of WES data from KD cases, a total of 902 control WES or whole genome sequencing
(WGS) data samples were obtained from Dr. Yeon-Mok Oh (Asan Medical Center, Seoul, Korea; WES data from 147 patients with chronic obstructive pulmonary disease), Dr. Byung-Ok Choi (Samsung Medical Center, Seoul, Korea; WES data from 258 patients with Charcot-Marie-Tooth disease), and the Biobank for Health Sciences (Center for Genome Science, Chungwon, Korea; WES data from 100 healthy subjects and WGS data from 397 healthy adults). For replication study, a total of 586 patients with complete $\mathrm{KD}$ were selected from our $\mathrm{KD}$ patient pool. The healthy adult control samples $(n=732)$ were obtained from the Biobank for Health Sciences (Chungwon, Korea). This study was approved by the institutional review board (IRB) at Asan Medical Center (IRB No. 2014-0823), and informed consent was obtained from the parents of patients with $\mathrm{KD}$.

\section{Targeted capture and exome sequencing}

WES was conducted using genomic DNA samples obtained from 200 children with $\mathrm{KD}$. For exome sequencing, $3 \mu \mathrm{g}$ genomic DNA was initially sheared into 150-base-pair (bp) fragments for shotgun library construction. Fragmented DNA from each sample was captured using the SureSelect Human All Exon V5 Kit (Agilent Technologies, Santa Clara, CA, USA), designed to cover $50 \mathrm{Mb}$ of human genomic sequences corresponding to the NCBI Consensus Coding Sequence database. Each captured library was sequenced at Macrogen (Seoul, Korea) using an Illumina HiSeq 2500 instrument (Illumina, San Diego, CA, USA), according to the manufacturer's instructions.

\section{Read mapping and variant analysis}

Sequence reads in FASTQ format were aligned to a human genome reference sequence (GRCh37) using Burrows-Wheeler Aligner (BWA) software [15]. Duplicate reads were marked in BAM files using Picard MarkDuplicates (https:// broadinstitute. github.io/picard/). Local realignment, base quality recalibration, variant calling, joint genotyping, and variant quality score recalibration and filtration were applied using Genome Analysis ToolKit (GATK) v3.7 (http:// software.broadinstitute.org/gatk/). Variant annotation was performed using SnpEff [16].

\section{Genotyping}

Genotyping for the replication study was performed on the high-throughput Fluidigm EP1 system (Fluidigm Corp., South San Francisco, CA, USA), using the Fluidigm SNPType assay platform, and nanofluidic 96.96 and 48.48 Dynamic Array Integrated Fluidic Circuits (Fluidigm). Genotype calls were made using the Fluidigm SNP Genotyping Analysis program (Fluidigm). All but 
one of the selected SNPs were genotyped for replication. The overall genotyping success rate was $99.5 \%$.

\section{SNP filtering}

After WES, a total of 870,062 SNPs were identified from $200 \mathrm{KD}$ cases and 902 controls. To filter the SNP markers, we excluded 337,282 SNP markers with missing call rates > 3\%, 138,329 monomorphic markers, and 3,404 multiallelic markers in patients with $\mathrm{KD}$. As coding variants that cause amino acid changes may be hypothesized to confer larger phenotypic effects, we also excluded 313,470 intergenic, intronic, untranslated-region, and synonymous SNPs. After filtering, 77,577 protein-altering variants remained, among which 75,365 were missense, 1,448 were nonsense, and 764 were in splice-acceptor/donor sites. Next, we excluded 3,209 SNPs with missing call rates > 5\% (slightly looser threshold due to heterogeneous control data compared to case data) in control data. Finally, a total of 74,368 SNPs were included in the association analysis.

\section{Statistical analysis}

All statistical analyses were performed using PLINK software v1.07 (http://pngu.mgh.harvard.edu/ purcell/plink/) [17]. The chi-square or Fisher's exact tests (when the expected cell count was less than five) were used to compare SNP allele frequencies between cases and controls to test for associations with $\mathrm{KD}$ and CAA.

\section{Results}

\section{Associations with $\mathrm{KD}$}

WES was initially performed using samples from 200 children with $\mathrm{KD}$, of which 159 had complete $\mathrm{KD}$ with a fever lasting $\geq 5$ days and $>4$ of the principal symptoms of $\mathrm{KD}$. Therefore, we performed association analysis of 159 complete $\mathrm{KD}$ cases and 902 controls. For the replication study, we selected 12 coding SNPs with significant associations with $\mathrm{KD}$ susceptibility (chi-square test $\mathrm{p}<0.001$ ) and immune-related functions. Extremely rare variants are more likely to be deleterious; thus we also selected an additional 51 immune-related rare coding SNPs detected in case samples only, or with odds ratios (ORs) $\geq 3$ in case-control comparisons. We tested these $63 \mathrm{SNPs}$ in 586 patients with complete $\mathrm{KD}$ and 732 healthy controls using the Fluidigm SNPType assay platform; however, none showed statistical significance $(\mathrm{p}>0.05)$ due to their very low frequencies in both case and control samples; however, among them, five coding SNPs (rs2275603 in FCRLA, rs755244149 in PTGER4, rs117796773 in IL17F, rs41493047 in

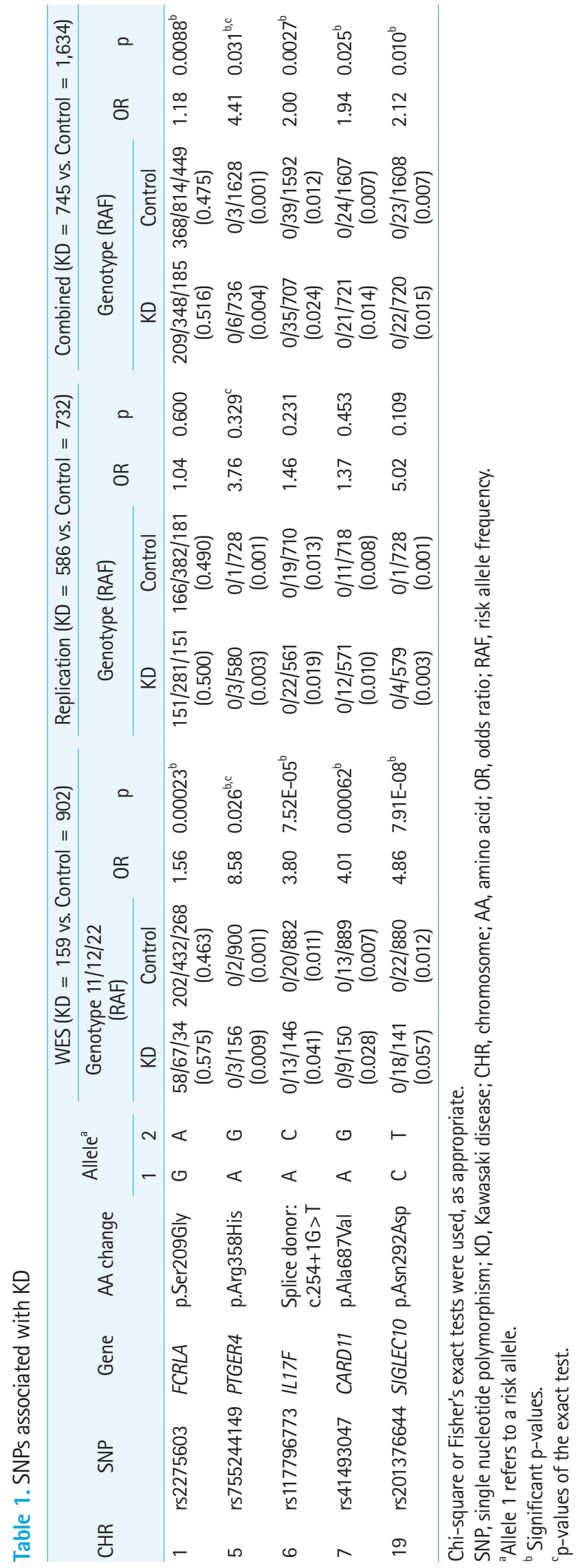


CARD11, and rs201376644 in SIGLEC10) showed nominal significances (OR, 1.18 to 4.41, $\mathrm{p}=0.0027-0.031)$ in combined analysis of data generated by WES and Fluidigm SNPType assay (Table 1).

\section{Associations with CAA}

Of the 200 children with $\mathrm{KD}, 74$ had coronary artery lesions. To investigate genetic loci that affect the development of CAAs in $\mathrm{KD}$, we initially stratified our $\mathrm{KD}$ samples by CAA size, according to the American Heart Association statement [13], as follows: small, 3 to $<5 \mathrm{~mm}$; medium, 5 to $8 \mathrm{~mm}$, or giant, $>8 \mathrm{~mm}$. Next, we performed association analysis in $124 \mathrm{KD}$ patients without CAAs $(<3 \mathrm{~mm}$ ) and $26 \mathrm{KD}$ patients with medium or giant aneurysms that showed more extreme phenotypes (internal lumen diameter of coronary arteries $>5 \mathrm{~mm}$ ). For the replication study, we selected five SNPs with significant associations with CAAs (chisquare test $\mathrm{p}<0.001$ ) and CAA-related functions, and tested them in 420 patients with $\mathrm{KD}$ without CAAs and 19 with CAAs (diameter $>5 \mathrm{~mm}$ ). Although no SNP showed significant results in the replication study, five coding variants (rs148721785 in IL$31 R A$, rs201812753 in FGFR4, rs374967242 in FNDC1, rs61754773 in MMP8, and rs188424977 in FOXN1) showed significant associations (OR, 3.89 to $37.3 ; \mathrm{p}=0.0058-0.0261)$ in combined analysis of the WES and replication samples (Table 2).

\section{Discussion}

Recent GWAS identified some clear KD-associated loci and successfully identified part of the genetic background of $\mathrm{KD}$; however, knowledge of these genetic factors has not made a significant contribution to understanding KD pathogenesis [12]. In this study, we performed WES with the aim of identifying rare protein-coding variants that contribute to KD susceptibility. We identified five rare coding variants in five genes (FCRLA, PTGER4, IL17F, CARD11, and SIGLEC10) associated with KD and another five coding variants in five genes (FGFR4, IL31RA, FNDC1, $M M P 8$, and FOXN1) associated with CAA.

Among the five $\mathrm{KD}$-associated genes, FCRLA has previously been reported to be highly significantly associated with low levels of affinity immunoglobulin gamma $\mathrm{Fc}$ region receptor II-a/b (products of FCGR2A and FCGR2B) in human blood (rs6668534: 0.434-unit decrease, $\mathrm{p}=6 \times 10^{-17}$ ) [18], as well as being associated with monocyte and lymphocyte counts [19]. FCGR2A is an established $\mathrm{KD}$ susceptibility gene $[7,10]$. These observations suggest that the effect of FCGR2A gene variants on KD susceptibility may be regulated by FCRLA. PTGER4 is one of

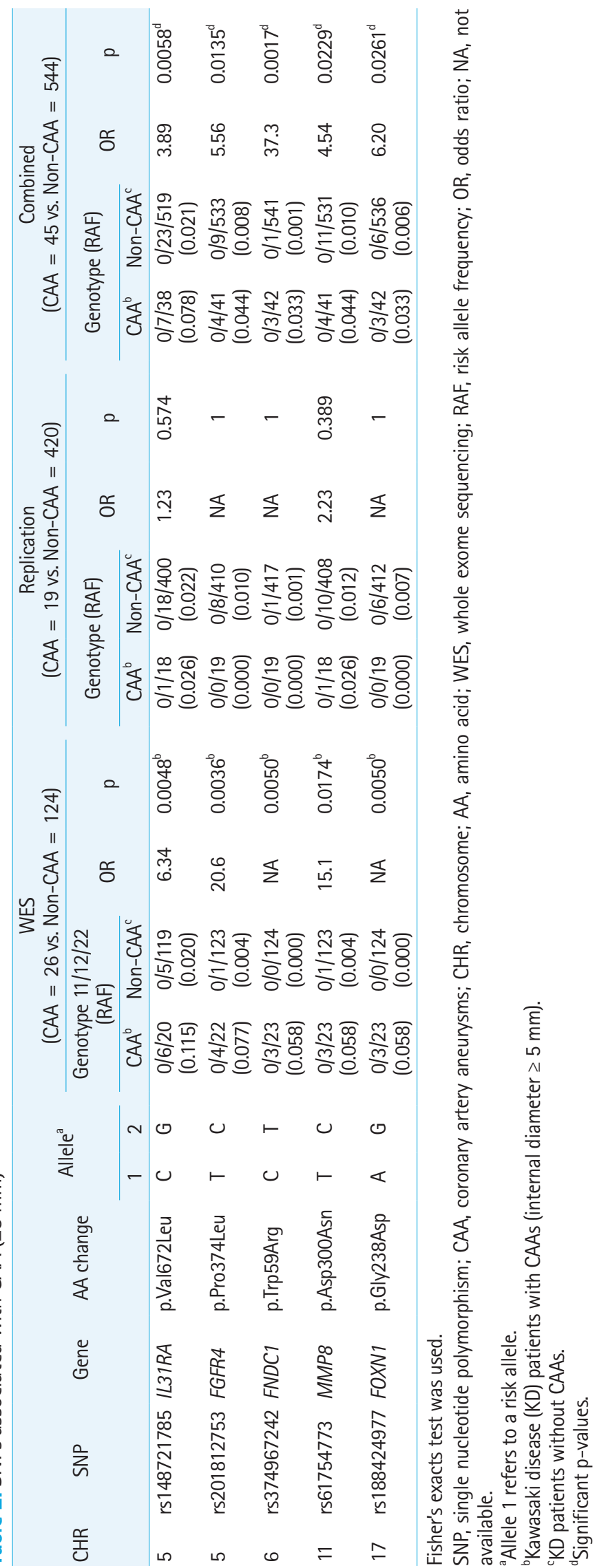


four identified receptors for prostaglandin E2 (PGE2). Previous studies investigated the function of PGE2 in relation to $\mathrm{KD}$ [2023]. PGE2 levels are markedly elevated during the acute stage of $\mathrm{KD}$ and then decrease during the recovery stage [20]. PGE2 level was also correlated with prevention of IVIG resistance and CAA formation through CD40L [23]. Thus, PTGER4, a PGE2 receptor, may function in $\mathrm{KD}$ pathogenesis. The role of the CARD11 gene in KD remains unknown; however, mutations of CARD11 can cause a condition referred to as 'B-cell expansion with $\mathrm{NF \kappa B}$ and $T$ cell anergy', and immunodeficiency 11 , due to defective B-cell receptor signaling $[24,25]$. CARD11 is also associated with various immune-related diseases and traits, including multiple sclerosis, eczema, and platelet count $[19,26,27]$. Furthermore, defective B-cell development and B-cell functions are well known to be involved in $\mathrm{KD}$ pathogenesis [28,29]. Therefore, CARD11 may play a role in susceptibility to KD. The roles of IL17F and SIGLEC10 in KD susceptibility are unknown.

To date, several GWAS have been performed to identify susceptibility loci for coronary artery formation in $\mathrm{KD}$, using a very limited number of patients with $\mathrm{KD}$ and CAA [30-34]. No overlapping genetic loci were detected from these GWAS. In this study, we identified five rare coding variants in five genes associated with CAAs in KD. None of these five genes, except for MMP8, have been reported to be associated with susceptibility to KD or CAAs in KD. MMP8 encodes a member of the matrix metalloproteinase (MMP) family of proteins, which may be involved in maintaining the structure and function of coronary vascular walls [35]. An increasing number of studies have indicated elevated expression, activity, or protein levels of MMPs in $\mathrm{KD}$ [36,37]. MMP8 expression levels are significantly upregulated in KD before IVIG treatment and decrease after undergoing IVIG treatment [37]. MMP8 is also highly abundant in IVIG-resistant $\mathrm{KD}$, suggesting that this is a potential biomarker for identifying those $\mathrm{KD}$ patients at highest risk for CAAs, and who may benefit from additional anti-inflammatory therapy $[38,39]$.

To our knowledge, this is the first WES study of $\mathrm{KD}$, and we identified several rare coding risk variants potentially associated with KD and CAA through WES and association analysis; however, we failed to confirm the significance of the selected SNPs in the replication study, mainly due to their extremely low frequency. Another reason for lack of significance in a replication study can be partly due to sample selection bias. To enrich for possible causative variants in the WES stage, we selected more severe KD cases than in the replication stage, such as patients with medium-sized or giant aneurysms (6.3\% in WES samples vs. $1.7 \%$ in replication samples), IVIG resistance ( $50.3 \%$ vs. $22.5 \%)$, recurrence ( $14.5 \%$ vs.
$5.5 \%)$, and family history (6.3\% vs. $1.9 \%)$. Therefore, more variants that result in these severe phenotypes may have been discovered in the WES stage; however, in combined analysis of WES and replication data, statistically significant associations were detected. WES is a powerful technique for identification of protein-coding variants, which are more straightforward to annotate for biological functions and help to pinpoint causative genes; however, functional variants are more likely to be rare. Therefore, large sample sizes are usually required to detect loci with exome-wide significance, particularly for highly polygenic traits; however, we were only able to sequence a small number of samples, particularly in the case of children with CAA. Both common and rare variant association approaches are drastically underpowered to detect associations in small samples. Thus, our conclusions are tentative and preliminary, and further studies with large sample size are necessary to confirm our findings, as well as the functional role of the newly identified genes and variants in $\mathrm{KD}$ pathogenesis.

In summary, we sequenced the whole exomes of patients with $\mathrm{KD}$ and identified five candidate genes (FCRLA, PTGER4, IL17F, CARD11, and SIGLEC10) that may influence KD susceptibility, and another five candidate genes (FGFR4, IL31RA, FNDC1, $M M P 8$, and FOXN1) with potential influence on the development of coronary aneurysms in $\mathrm{KD}$. These results provide insights into new candidate genes and genetic variants potentially involved in the development of $\mathrm{KD}$ and CAA. Further association studies with expanded $\mathrm{KD}$ sample numbers from the Korean population or from cohorts with different ethnicities are required to confirm these results.

\section{ORCID}

Jae-Jung Kim: https://orcid.org/0000-0002-7406-3261 Young Mi Hong: https://orcid.org/0000-0002-6600-7876 Sin Weon Yun: https://orcid.org/0000-0001-8947-703X Kyung-Yil Lee: https://orcid.org/0000-0001-6510-1580 Kyung Lim Yoon: https://orcid.org/0000-0003-4734-900X Myung-Ki Han: https://orcid.org/0000-0003-1817-8088 Gi Beom Kim: https://orcid.org/0000-0002-7880-280X Hong-Ryang Kil: https://orcid.org/0000-0003-4925-8240 Min Seob Song: https://orcid.org/0000-0003-3426-6803 Hyoung Doo Lee: https://orcid.org/0000-0002-6125-187X Kee Soo Ha: https://orcid.org/0000-0001-6753-5411 Hyun OkJun: https://orcid.org/0000-0003-2270-0123 Byung-Ok Choi: https://orcid.org/0000-0001-5459-1772 Yeon-Mok Oh: https://orcid.org/0000-0003-0116-4683 Jeong Jin Yu: https://orcid.org/0000-0003-1601-3685 
Gi Young Jang: https://orcid.org/0000-0002-4831-1188

Jong-Keuk Lee: https://orcid.org/0000-0003-1125-4017

\section{Authors' Contribution}

Conceptualization: JJY, GYJ, JKL. Data curation: JJK, YMH, SWY, KYL, KLY, MKH, GBK, HRK, MSS, HDL, KSH, HOJ, BOC, YMO, JJY, GYJ. Formal analysis: JJK. Funding acquisition: JKL. Methodology: JJK. Writing - original draft: JJK. Writing - review \& editing: JJY, GYJ, JKL.

\section{Conflicts of Interest}

No potential conflict of interest relevant to this article was reported.

\section{Acknowledgments}

We thank all of our patients and their families for participating in this study. This work was supported by a grant from the Ministry of Health \& Welfare of the Republic of Korea (HI15C1575) and a grant from the Korea Center for Disease Control and Prevention (2016-ER7401-00).

\section{References}

1. Burns JC, Glode MP. Kawasaki syndrome. Lancet 2004;364:533544.

2. Kato H, Sugimura T, Akagi T, Sato N, Hashino K, Maeno Y, et al. Long-term consequences of Kawasaki disease: a 10- to 21-year follow-up study of 594 patients. Circulation 1996;94:1379-1385 .

3. Newburger JW, Takahashi M, Burns JC, Beiser AS, Chung KJ, Duffy CE, et al. The treatment of Kawasaki syndrome with intravenous gamma globulin. N Engl J Med 1986;315:341-347.

4. Durongpisitkul K, Gururaj VJ, Park JM, Martin CF. The prevention of coronary artery aneurysm in Kawasaki disease: a meta-analysis on the efficacy of aspirin and immunoglobulin treatment. Pediatrics 1995;96:1057-1061.

5. Newburger JW, Fulton DR. Kawasaki disease. Curr Opin Pediatr 2004;16:508-514.

6. Lee KY, Han JW, Lee JS. Kawasaki disease may be a hyperimmune reaction of genetically susceptible children to variants of normal environmental flora. Med Hypotheses 2007;69:642-651.

7. Khor CC, Davila S, Breunis WB, Lee YC, Shimizu C, Wright VJ, et al. Genome-wide association study identifies FCGR2A as a susceptibility locus for Kawasaki disease. Nat Genet 2011;43:
1241-1246.

8. Lee YC, Kuo HC, Chang JS, Chang LY, Huang LM, Chen MR, et al. Two new susceptibility loci for Kawasaki disease identified through genome-wide association analysis. Nat Genet 2012;44: 522-525.

9. Onouchi Y, Ozaki K, Burns JC, Shimizu C, Terai M, Hamada H, et al. A genome-wide association study identifies three new risk loci for Kawasaki disease. Nat Genet 2012;44:517-521.

10. Kim JJ, Yun SW, Yu JJ, Yoon KL, Lee KY, Kil HR, et al. A genome-wide association analysis identifies NMNAT2 and HCP5 as susceptibility loci for Kawasaki disease. J Hum Genet 2017; 62:1023-1029.

11. Kwon YC, Kim JJ, Yun SW, Yu JJ, Yoon KL, Lee KY, et al. BCL2L11 is associated with Kawasaki disease in intravenous immunoglobulin responder patients. Circ Genom Precis Med 2018;11:e002020.

12. Onouchi Y. The genetics of Kawasaki disease. Int J Rheum Dis 2018;21:26-30.

13. Newburger JW, Takahashi M, Gerber MA, Gewitz MH, Tani LY, Burns JC, et al. Diagnosis, treatment, and long-term management of Kawasaki disease: a statement for health professionals from the Committee on Rheumatic Fever, Endocarditis and Kawasaki Disease, Council on Cardiovascular Disease in the Young, American Heart Association. Circulation 2004;110:2747-2771.

14. McCrindle BW, Rowley AH, Newburger JW, Burns JC, Bolger AF, Gewitz M, et al. Diagnosis, treatment, and long-term management of Kawasaki disease: a scientific statement for health professionals from the American Heart Association. Circulation 2017;135:e927-e999.

15. Li H, Durbin R. Fast and accurate short read alignment with Burrows-Wheeler transform. Bioinformatics 2009;25:1754-1760.

16. Cingolani P, Platts A, Wang le L, Coon M, Nguyen T, Wang L, et al. A program for annotating and predicting the effects of single nucleotide polymorphisms, SnpEff: SNPs in the genome of Drosophila melanogaster strain w1118; iso-2; iso-3. Fly (Austin) 2012;6:80-92.

17. Purcell S, Neale B, Todd-Brown K, Thomas L, Ferreira MA, Bender D, et al. PLINK: a tool set for whole-genome association and population-based linkage analyses. Am J Hum Genet 2007; 81:559-575.

18. Suhre K, Arnold M, Bhagwat AM, Cotton RJ, Engelke R, Raffler J, et al. Connecting genetic risk to disease end points through the human blood plasma proteome. Nat Commun 2017;8:14357.

19. Vuckovic D, Bao EL, Akbari P, Lareau CA, Mousas A, Jiang T, et al. The polygenic and monogenic basis of blood traits and diseases. Cell 2020;182:1214-1231. 
20. Lee T, Furukawa S, Fukuda Y, Yabuta K, Kato H. Plasma prostaglandin E2 level in Kawasaki disease. Prostaglandins Leukot Essent Fatty Acids 1988;31:53-57.

21. Kajimoto M, Ichiyama T, Ueno Y, Shiraishi M, Hasegawa M, Furukawa $S$. Enhancement of activated beta1-integrin expression by prostaglandin E2 via EP receptors in isolated human coronary arterial endothelial cells: implication for the treatment of Kawasaki disease. Inflamm Res 2009;58:224-228.

22. Sasai K. Plasma PGE2, TXB2 and 6-keto PGF1 alpha levels in patients with Kawasaki disease. Arerugi 1988;37:952-958.

23. Kuo HC, Wang CL, Yang KD, Lo MH, Hsieh KS, Li SC, et al. Plasma prostaglandin E2 levels correlated with the prevention of intravenous immunoglobulin resistance and coronary artery lesions formation via CD40L in Kawasaki disease. PLoS One 2016;11:e0161265.

24. Snow AL, Xiao W, Stinson JR, Lu W, Chaigne-Delalande B, Zheng L, et al. Congenital B cell lymphocytosis explained by novel germline CARD11 mutations. J Exp Med 2012;209:22472261.

25. Stepensky P, Keller B, Buchta M, Kienzler AK, Elpeleg O, Somech $\mathrm{R}$, et al. Deficiency of caspase recruitment domain family, member 11 (CARD11), causes profound combined immunodeficiency in human subjects. J Allergy Clin Immunol 2013;131:477485.

26. International Multiple Sclerosis Genetics Consortium; Beecham AH, Patsopoulos NA, Xifara DK, Davis MF, Kemppinen A, et al. Analysis of immune-related loci identifies 48 new susceptibility variants for multiple sclerosis. Nat Genet 2013;45:1353-1360.

27. Kichaev G, Bhatia G, Loh PR, Gazal S, Burch K, Freund MK, et al. Leveraging polygenic functional enrichment to improve GWAS power. Am J Hum Genet 2019; 104:65-75.

28. Yamazaki-Nakashimada MA, Gamez-Gonzalez LB, Murata C, Honda T, Yasukawa K, Hamada H. IgG levels in Kawasaki disease and its association with clinical outcomes. Clin Rheumatol 2019;38:749-754.

29. Lindquist ME, Hicar MD. B cells and antibodies in Kawasaki disease. Int J Mol Sci 2019;20:1834.
30. Kim JJ, Park YM, Yoon D, Lee KY, Seob Song M, Doo Lee H, et al. Identification of KCNN2 as a susceptibility locus for coronary artery aneurysms in Kawasaki disease using genome-wide association analysis. J Hum Genet 2013;58:521-525.

31. Kuo HC, Li SC, Guo MM, Huang YH, Yu HR, Huang FC, et al. Genome-wide association study identifies novel susceptibility genes associated with coronary artery aneurysm formation in Kawasaki disease. PLoS One 2016;11:e0154943.

32. Lin YJ, Chang JS, Liu X, Tsang H, Chien WK, Chen JH, et al. Genetic variants in PLCB4/PLCB1 as susceptibility loci for coronary artery aneurysm formation in Kawasaki disease in Han Chinese in Taiwan. Sci Rep 2015;5:14762.

33. Kwon YC, Kim JJ, Yu JJ, Yun SW, Yoon KL, Lee KY, et al. Identification of the TIFAB gene as a susceptibility locus for coronary artery aneurysm in patients with Kawasaki disease. Pediatr Cardiol 2019;40:483-488.

34. Hoggart C, Shimizu C, Galassini R, Wright VJ, Shailes H, Bellos E, et al. Identification of novel locus associated with coronary artery aneurysms and validation of loci for susceptibility to Kawasaki disease. Eur J Hum Genet 2021;29:1734-1744.

35. Hopps E, Caimi G. Matrix metalloproteases as a pharmacological target in cardiovascular diseases. Eur Rev Med Pharmacol Sci 2015; 19:2583-2589.

36. Senzaki H. The pathophysiology of coronary artery aneurysms in Kawasaki disease: role of matrix metalloproteinases. Arch Dis Child 2006;91:847-851.

37. Kuo HC, Li SC, Huang LH, Huang YH. Epigenetic hypomethylation and upregulation of matrix metalloproteinase 9 in Kawasaki disease. Oncotarget 2017;8:60875-60891.

38. Fury W, Tremoulet AH, Watson VE, Best BM, Shimizu C, Hamilton J, et al. Transcript abundance patterns in Kawasaki disease patients with intravenous immunoglobulin resistance. Hum Immunol 2010;71:865-873.

39. Pan Y, Fan Q. Identification of potential core genes in immunoglobulin-resistant Kawasaki disease using bioinformatics analysis. Crit Rev Eukaryot Gene Expr 2020;30:85-91. 